\title{
Effect of cylinder power and axis changes on vision in astigmatic participants
}

This article was published in the following Dove Medical Press journal:

Clinical Optometry

\author{
J Sha' \\ C Fedtke ${ }^{1,2}$ \\ D Tilia ${ }^{1,2}$ \\ N Yeotikar' \\ $M$ Jong ${ }^{1,2}$ \\ J Diec' \\ V Thomas' \\ RC Bakaraju ${ }^{1,2}$
}

'Brien Holden Vision Institute, Sydney, NSW, Australia; ${ }^{2}$ School of Optometry and Vision Science, University of New South Wales, Sydney, NSW, Australia
Correspondence: RC Bakaraju Brien Holden Vision Institute, Level 5, Rupert Myers Building, North Wing Gate I4 Barker Street, Sydney, NSW 2052, Australia

Tel +61293857516

Fax +61293857401

Email r.bakaraju@brienholdenvision.org
Purpose: To ascertain the impact of altering cylinder (cyl) power and axis on vision in astigmatism.

Methods: In a prospective, randomized, participant-masked, crossover clinical trial, 28 astigmatic participants were tested for the following conditions on different days: full sphero-cyl correction and undercorrection by $0.25,0.50$, and $0.75 \mathrm{DC}$ while maintaining spherical equivalence. Axis was also misaligned between $-30^{\circ}$ and $+30^{\circ}$, in $10^{\circ}$ steps. For each configuration, monocular high- and low-contrast visual acuities (HCVA, LCVA) were measured at $6 \mathrm{~m}$, and participants rated vision clarity (1-10), vision satisfaction (1-10), and vision acceptability (yes/ no). Linear mixed models were used to compare visual performance in the overall group and in low, medium, and high cyl subgroups.

Results: Undercorrecting cyl power affected all groups equally $(P \geq 0.073)$. Undercorrection by $0.75 \mathrm{DC}$ was significantly different to full cyl power for all variables $(P \leq 0.007)$, while 0.25 DC undercorrection did not cause any significant decreases $(P>0.05)$. Undercorrection by 0.50 DC was significantly different to full cyl power for HCVA $(P=0.006$, however not clinically significant $)$ and vision acceptability $(P=0.034)$. Axis misalignment affected the cyl groups differently $(P<0.001)$, with the greatest impact in the high cyl group, followed by the medium then the low-cyl group. Misalignment by $\pm 30^{\circ}$ caused significant decreases in almost all cases ( $P \leq 0.003$ ), while misalignments by $\pm 10^{\circ}$ or $\pm 20^{\circ}$ caused significant decreases for some cyl groups and test variables.

Conclusion: Undercorrection of cyl by $\leq 0.50 \mathrm{DC}$ while maintaining spherical equivalence has no significant effect on HCVA, LCVA, vision clarity, and vision satisfaction, while the amount of axis misalignment that can be tolerated is dependent on the cyl power. These results may have practical ophthalmic applications, such as reducing the total number of stock keeping units of toric contact lenses.

Keywords: astigmatism, toric, sensitivity, misalignment, visual performance

\section{Introduction}

Astigmatism is a common refractive error resulting from a toric-surfaced cornea and/ or a toric-surfaced, tilted or decentered crystalline lens. Possible causes of astigmatism include genetics, ${ }^{1,2}$ eyelid and extraocular muscle anatomy, ${ }^{3-6}$ ocular conditions such as keratoconus, ${ }^{7}$ and abnormalities during emmetropization. ${ }^{8,9}$ The prevalence of at least $0.75 \mathrm{DC}$ of astigmatism has been reported in various populations, ${ }^{10-19}$ ranging from $6.9 \%$ in $\leq 15$-year-old patients in Southern India $^{10}$ to $73.5 \%$ in $>15$-year-old patients in central Europe. ${ }^{11}$ One UK study estimated that a third of potential contact lens wearers require astigmatic correction, ${ }^{16}$ and in $2016,22 \%$ of soft contact lenses 
prescribed internationally were toric. ${ }^{20}$ Apart from soft toric contact lenses, other forms of astigmatism correction include spectacles, rigid contact lenses, and refractive surgery.

Due to the numerous possible combinations between sphere powers, cylinder powers, and cylinder axes, manufacturers of disposable soft toric contact lenses limit their toric lens range, while still aiming to cater for the majority of astigmatic patients. Depending on the brand and manufacturer, these toric lenses usually comprise $3-5$ cylinder powers and 10-18 cylinder axes for each sphere power (which itself may include 40-60 powers). Despite limiting the toric parameters, it is clear that this still represents an immense number of stock-keeping units (SKUs), both for the manufacturers and for the eye-care practitioners who wish to have trial contact lenses on hand in their practice.

In fact, efforts are being made to achieve contact lens correction of astigmatism that is independent of lens axis using a bifocal design ${ }^{21}$ which would vastly reduce the number of required SKUs. However, this technology may still be several years away and may not be suitable for all toric contact lens wearers, as approximately a third of participants wearing this lens noticed constant double images. ${ }^{21}$

Previous studies have reported on the effect of uncorrected or misaligned cylinder on vision. ${ }^{22-30}$ Many of these used model eyes or induced astigmatism at specified powers and axes which may have been different to the natural cylinder of the eye. To the best of our knowledge, no study to date has evaluated the effects of undercorrecting cylinder power and misaligning cylinder axis in amounts relevant to toric contact lenses, as well as their interactions, in human eyes based on their natural cylinder. Hence, in order to understand whether it is feasible to reduce the number of soft toric contact lens SKUs, the aim of this study was to determine the degree to which altering cylinder power and axis can impact visual performance in people with astigmatism. These findings may also assist eye-care practitioners in situations where an ideal toric lens power or axis is not available, or when a toric lens is unstable or misaligning.

\section{Methods}

\section{Design}

This was a prospective, participant-masked, cross-over, randomized clinical trial conducted at the Clinical Research Trials Center (CRTC) of the Brien Holden Vision Institute in Sydney, Australia. A Human Research Ethics Committee (Bellberry, Adelaide, South Australia) approved the clinical trial. Written informed consent from each participant was obtained prior to commencing any study procedures. The clinical trial conformed to the principles of the Declaration of Helsinki and was registered on the Australian New Zealand Clinical Trials Registry (ACTRN12616001526460).

\section{Participants}

The inclusion criteria for the study were as follows: aged 18 years or older, having $\geq 0.75 \mathrm{DC}$ in at least one eye, and having ocular and systemic health that would not prevent accurate visual acuity measurements.

\section{Procedures}

Baseline visits determined participants' subjective distance refraction using standard optometric techniques. Monocular high- and low-contrast visual acuity (HCVA and LCVA) were measured using an electronic logMAR letter chart, Test Chart 2000 Pro (Thompson Software Solutions, Hertfordshire, UK), at $6 \mathrm{~m}$ with contrast set to $100 \%$ and $10 \%$, respectively, with a room illumination of 350-400 1x. Participants' baseline higher-order aberrations (up to fourth order) were measured using the BHVI-EyeMapper ${ }^{31}$ (Brien Holden Vision Institute, Sydney, Australia) in low illumination $(\sim 10 \mathrm{~lx})$ under their natural pupil.

Following the baseline visit, participants attended four assessment visits (Figure 1). The first could be performed immediately after the baseline visit, but otherwise each was on a separate day. Participants were required to wear spectacles on the days of the assessment visits. At each assessment visit, a different cylinder power was tested: 1) full cylinder power, 2) undercorrection of cylinder power by $0.25 \mathrm{DC}, 3$ ) undercorrection of cylinder power by $0.50 \mathrm{DC}$ (sphere power compensated by $-0.25 \mathrm{D}$ ) or 4 ) undercorrection of cylinder power by $0.75 \mathrm{DC}$ (sphere power compensated by $-0.25 \mathrm{D}$ ). The order of these powers was randomized to the assessment visits, and the same randomization was applied to both eyes.

For each of the four cylinder powers, the following cylinder axes were tested monocularly for both eyes: 1) the correct axis, 2) misaligned by $+10^{\circ}, 3$ ) misaligned by $+20^{\circ}$, 4) misaligned by $\left.+30^{\circ}, 5\right)$ misaligned by $-10^{\circ}$,6) misaligned by $-20^{\circ}$, and 7) misaligned by $-30^{\circ}$. The order of these axes was randomized and applied to both eyes. Positive and negative misalignments were always in the anticlockwise and clockwise directions, respectively.

The changes to cylinder power and axes were made using a standard optometric phoropter (Inami L-7040, Tokyo, Japan or Topcon VT-10, Tokyo, Japan). With each combination of power and axis, monocular HCVA and LCVA were measured at $6 \mathrm{~m}$. Participants were initially presented with nine lines of five letters ranging from $0.6 \log$ MAR $(6 / 24)$ to -0.2 


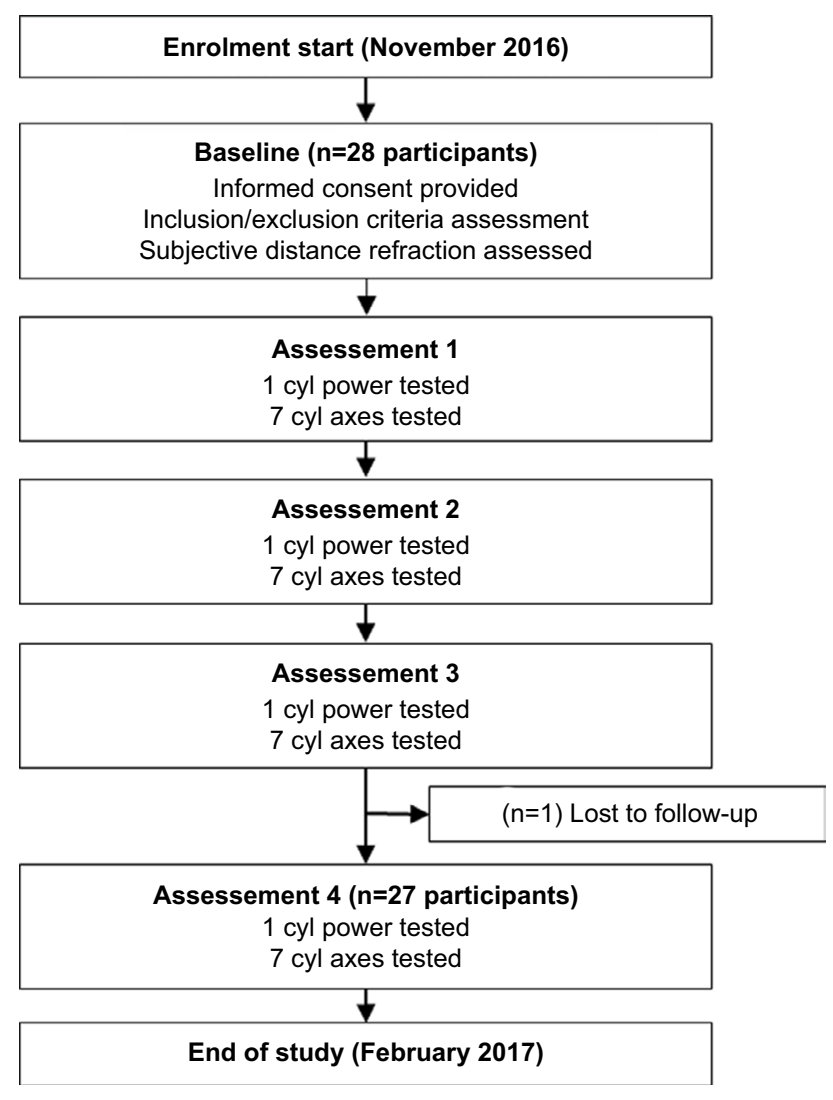

Figure I Participant flowchart. Abbreviation: cyl, cylinder.

$\log$ MAR (6/3.8) and instructed to read the lowest line that they could. If participants read the line correctly, they were encouraged to read the next line below, until they read at least three letters $(0.06 \log \mathrm{MAR})$ incorrectly on a line. Letters on the chart were randomized every time cylinder power and/or axis were changed. Participants also rated monocularly the clarity of the high-contrast $0.0 \log \mathrm{MAR}(6 / 6)$ line at $6 \mathrm{~m}$ from 1 to $10(1=$ blurred, $10=$ clear $)$, their satisfaction of vision of the whole high-contrast chart (bottom line $=6 / 6$ ) from 1 to $10(1=$ not satisfied, $10=$ satisfied $)$, and if their vision of the whole high-contrast chart was acceptable (yes/no).

All visual performance measurements were performed under photopic conditions ( $350-400 \mathrm{~lx})$. Instances for which a participant's cylinder power in one eye was less than the assigned undercorrection (eg, participant has $0.50 \mathrm{DC}$ in his or her right eye, assigned to be undercorrected by 0.75 DC), no measurements were taken for that eye at that visit. In instances where a participant's cylinder power was equal to the assigned undercorrection (eg, participant has 0.75 DC in his or her right eye, assigned to be undercorrected by $0.75 \mathrm{DC}$ ), only on-axis measurements were taken for that eye at that visit.

\section{Statistical analysis}

A minimum sample of 20 eyes was required to demonstrate a statistically significant paired difference of $1.0 \pm 1.5$ units in subjective ratings and $0.10 \pm 0.15 \log$ MAR in visual acuity at the $5 \%$ level of significance with $80 \%$ power.

Data were summarized as means \pm SD for variables measured on an interval scale and as percentages for categorical variables. A linear mixed model with subject random intercepts was deployed to test the hypothesis that changes to cylinder power and axis had an effect on visual acuity and subjective vision. The linear mixed model accounted for within subject correlation due to eye-specific data. The model included the following factors: amount of cylinder undercorrection, amount of cylinder axis misalignment, and cylinder group (low cylinder power $\leq 0.75 \mathrm{DC}$, medium cylinder power $1.00 \mathrm{DC}-1.75 \mathrm{DC}$, and high cylinder power $\geq 2.00 \mathrm{DC}$ ). The interactions of cylinder group with the other two factors were tested, and if significant, further analyses were performed to determine the significance of those factors within sublevels of cylinder groups. Vision acceptability was analyzed using logistic regression and chi-squared test. Post hoc multiple comparisons were corrected using Bonferroni correction. All the analyses were performed using SPSS 21 (IBM, Armonk, NY, USA).

\section{Results}

A total of 28 participants were enrolled in the study, of whom $57 \%$ were females, the mean \pm SD age was $45 \pm 15$ years (range 19-73 years), 61\% were Caucasian, 28\% were Asian, and $11 \%$ were of other ethnicity. The distribution of cylinder powers were as follows: $28.6 \%$ of eyes $(n=16)$ with low cylinder, $57.1 \%(\mathrm{n}=32)$ with medium cylinder, and $14.3 \%$ $(n=8)$ with high cylinder. Subjective refraction data from the subgroups and the overall sample are included in Table 1. With-the-rule and against-the-rule astigmatism were defined as axes within $15^{\circ}$ of $180^{\circ}$ and $90^{\circ}$, respectively. All other axes were considered oblique. One participant of the high cylinder group was lost to follow-up after completing three out of four assessment visits. This participant's data prior to discontinuation were included in the analysis. Additionally, it was necessary to exclude one medium cylinder participant's data from one assessment visit in the analysis due to a software error. Overall, the low-cylinder group achieved the best visual performance followed by the medium cylinder group then the high cylinder group. For example, mean \pm SD vision satisfaction ratings with full cylinder correction (power and axis) were 9.1 $\pm 1.4,7.8 \pm 2.1$, and $7.1 \pm 2.6$ in the low, medium, and high cylinder groups, respectively. 


\section{Effect of cylinder power}

The results of altering cylinder power while keeping the correct axis are presented in Table 2. At the correct axis, cylinder undercorrection and cylinder group were significant factors for all variables $(P \leq 0.027)$ except vision acceptability $(P \geq 0.681)$. The interaction of cylinder undercorrection and cylinder group was not significant for any variable $(P \geq 0.073)$, suggesting that undercorrecting cylinder power affected all groups equally overall. Post hoc analysis showed that the effect of undercorrecting cylinder power was mainly driven by undercorrection by $0.75 \mathrm{DC}(P \leq 0.007)$ for all variables.
Undercorrection by $0.50 \mathrm{DC}$ was significantly different to full cylinder power for HCVA $(P=0.006)$, however, not clinically significant, ${ }^{32}$ and vision acceptability $(P=0.034)$. Undercorrection by $0.25 \mathrm{DC}$ did not cause any significant decreases in visual performance $(P>0.05)$.

\section{Effect of cylinder axis}

The results of altering cylinder axis while keeping the correct cylinder power correction are presented in Table 3. At full cylinder power, axis misalignment and cylinder group were significant factors for all variables $(P<0.001)$ except vision

Table I Mean \pm SD subjective sphere power, cylinder power, M (spherical equivalent), J (WTR/ATR astigmatism component), $\mathrm{J}_{45}$ (OBL astigmatism component), and proportion of WTR:ATR:OBL in the low ( $\leq 0.75 \mathrm{DC})$, medium (1.00-1.75 DC), and high ( $\geq 2.00$ DC) cylinder groups, and in the overall sample.

\begin{tabular}{|l|l|l|l|l|}
\hline Variable & Low cylinder $(\mathbf{n}=\mathbf{1 6})$ & Medium cylinder $(\mathbf{n}=\mathbf{3 2})$ & High cylinder $(\mathbf{n}=\mathbf{8})$ & Overall sample $(\mathbf{n}=\mathbf{5 6})$ \\
\hline Subjective sphere (D) & $-3.05 \pm 2.66$ & $-1.86 \pm 2.91$ & $1.25 \pm 4.18$ & $-1.75 \pm 3.27$ \\
\hline Subjective cylinder (DC) & $-0.69 \pm 0.14$ & $-1.32 \pm 0.26$ & $-2.88 \pm 1.32$ & $-1.36 \pm 0.86$ \\
\hline M (D) & $-3.39 \pm 2.66$ & $-2.52 \pm 2.86$ & $-0.19 \pm 3.86$ & $-2.44 \pm 3.07$ \\
\hline$J_{0}(D)$ & $0.03 \pm 0.31$ & $-0.08 \pm 0.60$ & $0.26 \pm 1.47$ & $0.003 \pm 0.72$ \\
\hline$J_{45}(D)$ & $0.02 \pm 0.19$ & $-0.03 \pm 0.31$ & $-0.09 \pm 0.75$ & $-0.02 \pm 0.37$ \\
\hline WTR:ATR:OBL (\%) & $44: 25: 31$ & $38: 47: 16$ & $38: 50: 13$ & $39: 41: 20$ \\
\hline
\end{tabular}

Abbreviations: ATR, against-the-rule; OBL, oblique; WTR, with-the-rule.

Table 2 Effect of undercorrecting cylinder power while keeping cylinder axis aligned for the study variables in the low ( $\leq 0.75 \mathrm{DC})$, medium ( $1.00-1.75 \mathrm{DC})$, and high ( $\geq 2.00 \mathrm{DC})$ cylinder groups, and in the overall sample.

\begin{tabular}{|c|c|c|c|c|c|}
\hline Variable & & $\begin{array}{l}\text { Low cylinder } \\
(n=16)^{\mathrm{a}}\end{array}$ & $\begin{array}{l}\text { Medium cylinder } \\
(n=32)\end{array}$ & $\begin{array}{l}\text { High cylinder } \\
(n=8)\end{array}$ & $\begin{array}{l}\text { Overall sample } \\
(n=56)\end{array}$ \\
\hline HCVA (logMAR) & $\begin{array}{l}\text { Full cylinder power } \\
\text { Undercorrection by } 0.25 \mathrm{DC} \\
\text { Undercorrection by } 0.50 \mathrm{DC} \\
\text { Undercorrection by } 0.75 \mathrm{DC}\end{array}$ & $\begin{array}{l}-0.10 \pm 0.08 \\
-0.05 \pm 0.06 \\
-0.08 \pm 0.07 \\
-0.04 \pm 0.07\end{array}$ & $\begin{array}{l}-0.07 \pm 0.07 \\
-0.06 \pm 0.06 \\
-0.03 \pm 0.05 \\
0.02 \pm 0.07\end{array}$ & $\begin{array}{l}-0.003 \pm 0.13 \\
0.03 \pm 0.11 \\
0.06 \pm 0.16 \\
0.02 \pm 0.17\end{array}$ & $\begin{array}{l}-0.07 \pm 0.09 \\
-0.04 \pm 0.07 \\
-0.03 \pm 0.09 \\
0.001 \pm 0.09\end{array}$ \\
\hline LCVA (logMAR) & $\begin{array}{l}\text { Full cylinder power } \\
\text { Undercorrection by } 0.25 \mathrm{DC} \\
\text { Undercorrection by } 0.50 \mathrm{DC} \\
\text { Undercorrection by } 0.75 \mathrm{DC}\end{array}$ & $\begin{array}{l}0.22 \pm 0.12 \\
0.29 \pm 0.12 \\
0.26 \pm 0.13 \\
0.32 \pm 0.17\end{array}$ & $\begin{array}{l}0.27 \pm 0.20 \\
0.27 \pm 0.09 \\
0.30 \pm 0.13 \\
0.35 \pm 0.12\end{array}$ & $\begin{array}{l}0.32 \pm 0.15 \\
0.33 \pm 0.15 \\
0.37 \pm 0.20 \\
0.34 \pm 0.19\end{array}$ & $\begin{array}{l}0.26 \pm 0.17 \\
0.28 \pm 0.11 \\
0.30 \pm 0.15 \\
0.34 \pm 0.14\end{array}$ \\
\hline Vision clarity $(\mathrm{I}-\mathrm{I} 0)$ & $\begin{array}{l}\text { Full cylinder power } \\
\text { Undercorrection by } 0.25 \mathrm{DC} \\
\text { Undercorrection by } 0.50 \mathrm{DC} \\
\text { Undercorrection by } 0.75 \mathrm{DC}\end{array}$ & $\begin{array}{l}8.9 \pm 1.6 \\
7.9 \pm 2.0 \\
8.6 \pm 1.9 \\
7.2 \pm 2.0\end{array}$ & $\begin{array}{l}7.5 \pm 2.2 \\
7.5 \pm 2.0 \\
7.0 \pm 2.2 \\
5.0 \pm 2.3\end{array}$ & $\begin{array}{l}5.1 \pm 2.1 \\
5.4 \pm 2.4 \\
3.8 \pm 2.7 \\
4.5 \pm 2.5\end{array}$ & \begin{tabular}{|l|}
$7.6 \pm 2.3$ \\
$7.3 \pm 2.2$ \\
$6.9 \pm 2.6$ \\
$5.5 \pm 2.4$
\end{tabular} \\
\hline Vision satisfaction $(I-I 0)$ & $\begin{array}{l}\text { Full cylinder power } \\
\text { Undercorrection by } 0.25 \mathrm{DC} \\
\text { Undercorrection by } 0.50 \mathrm{DC} \\
\text { Undercorrection by } 0.75 \mathrm{DC}\end{array}$ & $\begin{array}{l}9.1 \pm 1.4 \\
8.5 \pm 1.6 \\
8.7 \pm 1.8 \\
7.6 \pm 2.2\end{array}$ & $\begin{array}{l}7.8 \pm 2.1 \\
8.1 \pm 1.9 \\
7.4 \pm 2.3 \\
6.0 \pm 1.9\end{array}$ & $\begin{array}{l}7.1 \pm 2.6 \\
7.3 \pm 2.4 \\
7.0 \pm 2.4 \\
6.3 \pm 1.6\end{array}$ & $\begin{array}{l}8.1 \pm 2.1 \\
8.1 \pm 1.9 \\
7.7 \pm 2.2 \\
6.4 \pm 2.0\end{array}$ \\
\hline Vision acceptability (yes \%) & $\begin{array}{l}\text { Full cylinder power } \\
\text { Undercorrection by } 0.25 \mathrm{DC} \\
\text { Undercorrection by } 0.50 \mathrm{DC} \\
\text { Undercorrection by } 0.75 \mathrm{DC}\end{array}$ & $\begin{array}{l}93.8 \%(15 / 16) \\
81.3 \%(13 / 16) \\
86.7 \%(13 / 15) \\
84.6 \%(11 / 13)\end{array}$ & \begin{tabular}{|l|}
$93.8 \%(30 / 32)$ \\
$90.0 \%(27 / 30)$ \\
$68.8 \%(22 / 32)$ \\
$59.4 \%(19 / 32)$
\end{tabular} & $\begin{array}{l}87.5 \%(7 / 8) \\
87.5 \%(7 / 8) \\
62.5 \%(5 / 8) \\
66.7 \%(4 / 6)\end{array}$ & \begin{tabular}{|l|}
$92.9 \%(52 / 56)$ \\
$87.0 \%(47 / 54)$ \\
$72.7 \%(40 / 55)$ \\
$66.7 \%(34 / 51)$ \\
\end{tabular} \\
\hline
\end{tabular}

Notes: Shaded values indicate a statistically significant difference to the full cylinder power from post hoc analysis $(P \leq 0.05)$. Bold values indicate where the difference is also clinically significant. ${ }^{a} n$ varies as required undercorrection was greater than some participants' required cylinder in one or both eyes.

Abbreviations: HCVA, high-contrast visual acuities; LCVA, low-contrast visual acuities. 
Table 3 Effect of misaligning cylinder axis by $\pm 10, \pm 20$, and \pm 30 while keeping the full cylinder power in the low ( $\leq 0.75 \mathrm{DC})$, medium (I.00-1.75 DC), and high ( $\geq 2.00 \mathrm{DC})$ cylinder groups and in the overall sample.

\begin{tabular}{|c|c|c|c|c|c|}
\hline \multicolumn{2}{|l|}{ Variable } & \multirow{2}{*}{$\begin{array}{l}\text { Low cylinder } \\
(n=16)\end{array}$} & \multirow{2}{*}{$\begin{array}{l}\text { Medium cylinder } \\
(\mathbf{n}=32)\end{array}$} & \multirow{2}{*}{$\begin{array}{l}\text { High cylinder } \\
(\mathbf{n}=8)\end{array}$} & \multirow{2}{*}{$\begin{array}{l}\begin{array}{l}\text { Overall sample } \\
(n=56)\end{array} \\
-0.07 \pm 0.09\end{array}$} \\
\hline HCVA (logMAR) & Axis aligned & & & & \\
\hline & Misalignment by \pm 10 & $-0.04 \pm 0.14$ & $-0.02 \pm 0.10$ & $0.11 \pm 0.14$ & $-0.01 \pm 0.13$ \\
\hline & Misalignment by \pm 20 & $-0.04 \pm 0.10$ & $0.04 \pm 0.10$ & $0.26 \pm 0.19$ & $0.05 \pm 0.15$ \\
\hline & Misalignment by \pm 30 & $-0.02 \pm 0.09$ & $0.14 \pm 0.11$ & $0.35 \pm 0.23$ & $0.12 \pm 0.17$ \\
\hline \multirow[t]{4}{*}{ LCVA (logMAR) } & Axis aligned & $0.22 \pm 0.12$ & $0.27 \pm 0.20$ & $0.32 \pm 0.15$ & $0.26 \pm 0.17$ \\
\hline & Misalignment by \pm 10 & $0.25 \pm 0.14$ & $0.30 \pm 0.19$ & $0.40 \pm 0.15$ & $0.30 \pm 0.18$ \\
\hline & Misalignment by \pm 20 & $0.28 \pm 0.13$ & $0.36 \pm 0.13$ & $0.57 \pm 0.20$ & $0.37 \pm 0.17$ \\
\hline & Misalignment by \pm 30 & $0.29 \pm 0.11$ & $0.45 \pm 0.14$ & $0.69 \pm 0.25$ & $0.44 \pm 0.20$ \\
\hline \multirow[t]{4}{*}{ Vision clarity $(\mathrm{I}-\mathrm{I} 0)$} & Axis aligned & $8.9 \pm 1.6$ & $7.5 \pm 2.2$ & $5.1 \pm 2.1$ & $7.6 \pm 2.3$ \\
\hline & Misalignment by \pm 10 & $8.0 \pm 2.4$ & $6.3 \pm 2.5$ & $2.9 \pm 1.4$ & $6.3 \pm 2.8$ \\
\hline & Misalignment by \pm 20 & $8.0 \pm 2.2$ & $4.6 \pm 2.4$ & $1.4 \pm 0.7$ & $5.1 \pm 3.1$ \\
\hline & Misalignment by \pm 30 & $7.0 \pm 2.1$ & $2.6 \pm 1.8$ & $1.1 \pm 0.5$ & $3.7 \pm 2.8$ \\
\hline \multirow{4}{*}{$\begin{array}{l}\text { Vision satisfaction } \\
(I-10)\end{array}$} & Axis aligned & $9.1 \pm 1.4$ & $7.8 \pm 2.1$ & $7.1 \pm 2.6$ & $8.1 \pm 2.1$ \\
\hline & Misalignment by \pm 10 & $8.4 \pm 2.4$ & $7.0 \pm 2.2$ & $6.3 \pm 1.9$ & $7.3 \pm 2.3$ \\
\hline & Misalignment by \pm 20 & $8.5 \pm 2.0$ & $5.3 \pm 2.4$ & $3.9 \pm 2.0$ & $6.0 \pm 2.8$ \\
\hline & Misalignment by \pm 30 & $7.8 \pm 2.0$ & $3.7 \pm 1.9$ & $2.8 \pm 1.8$ & $4.7 \pm 2.7$ \\
\hline \multirow{4}{*}{$\begin{array}{l}\text { Vision acceptability } \\
\text { (yes \%) }\end{array}$} & Axis aligned & $93.8 \%(15 / 16)$ & $93.8 \%(30 / 32)$ & $87.5 \%(7 / 8)$ & $92.9 \%(52 / 56)$ \\
\hline & Misalignment by \pm 10 & $87.5 \%(28 / 32)$ & $79.7 \%(51 / 64)$ & $43.8 \%(7 / 16)$ & $76.8 \%(86 / I I 2)$ \\
\hline & Misalignment by \pm 20 & $87.5 \%(28 / 32)$ & $40.6 \%(26 / 64)$ & $12.5 \%(2 / 16)$ & $50.0 \%(56 / I I 2)$ \\
\hline & Misalignment by \pm 30 & $87.5 \%(28 / 32)$ & $9.4 \%(6 / 64)$ & $12.5 \%(2 / 16)$ & $32.1 \%(36 / I I 2)$ \\
\hline
\end{tabular}

Notes: Shaded values indicate a significant difference to the correct axis from post hoc analysis $(P \leq 0.05)$. Bold values indicate where the difference is also clinically significant. Abbreviations: HCVA, high-contrast visual acuities; LCVA, low-contrast visual acuities.

acceptability $(P \geq 0.660)$. The interaction of axis misalignment and cylinder group was significant for all variables $(P<0.001)$, suggesting that axis misalignment affected the cylinder groups differently. Observation of the mean values shows, in general, the greatest impact in the high cylinder group, followed by the medium cylinder group and then the low cylinder group. HCVA appeared to be the most sensitive variable to axis misalignment, with misalignment by $\pm 10^{\circ}$ or more causing a significant decrease for all cylinder groups $(P \leq 0.042)$, though not always by a clinically significant amount. Misalignment by $\pm 30^{\circ}$ caused a significant decrease in all study variables in the overall sample and all cylinder groups $(P \leq 0.003)$ except vision acceptability in the low cylinder group $(P=0.914)$, while misalignments by $\pm 10^{\circ}$ or $\pm 20^{\circ}$ caused significant decreases for some cylinder groups and test variables (Table 3 ).

\section{Cylinder power and axis interaction}

Results of all cylinder power and axis combinations are shown in Figures 2-6. The decreased peak performance and the increased steepness of slope with increasing cylinder group can be seen for each variable.

\section{Higher-order aberrations}

The mean \pm SD pupil size in the low, medium, and high cylinder groups were 5.91 $\pm 0.84,5.70 \pm 0.80$, and $5.38 \pm 0.70 \mathrm{~mm}$, respectively, which were not significantly different between the groups $(P=0.908)$. Aberrations were measured in low illumination using a 4-mm pupil size to better approximate conditions under which visual acuity and subjective responses were performed. No significant differences in higher-order aberrations were found between cylinder groups $(P>0.05)$ including the root mean square values for higher-order aberrations $(0.02 \pm 0.01,0.02 \pm 0.01$, and $0.02 \pm 0.004$ for the low, medium, and high cylinder groups, respectively, $P=0.756$ ).

\section{Discussion}

The purpose of this study was to ascertain the degree to which altering cylinder power and axis can impact visual acuity and subjective vision responses in people with astigmatism, in order to understand the feasibility of reducing the number of SKUs of soft toric contact lenses. The results from the current study show that undercorrecting cylinder by 0.25 or $0.50 \mathrm{DC}$ while maintaining spherical equivalence was not different to the required cylinder power for HCVA, LCVA, 
vision clarity ratings, and vision satisfaction ratings (though undercorrection by $0.50 \mathrm{DC}$ was significantly different to full correction for HCVA, the difference was only two letters hence not clinically significant). ${ }^{32}$ However, undercorrecting by 0.75 DC resulted in worse HCVA, LCVA, vision clarity, vision satisfaction, and vision acceptability. This supports evidence that astigmatism $<0.50 \mathrm{DC}$ does not degrade visual acuity and correcting is of limited benefit, ${ }^{33}$ while correcting astigmatism of $0.75 \mathrm{DC}$ or more results in significantly better acuity than the spherical equivalent. ${ }^{34,35}$ On the other hand, Pujol et al reported that the greatest decrease in retinal image quality occurs when going from full cylinder correction to $0.25 \mathrm{DC}$ of undercorrection. ${ }^{26}$ Again, this is not reflective of the results from the current study, where 0.25 DC of undercorrection was tolerated quite well. We note that their results were based on just three eyes with relatively low amounts of astigmatism, and measuring the modulation transfer function via the double-pass technique might not be
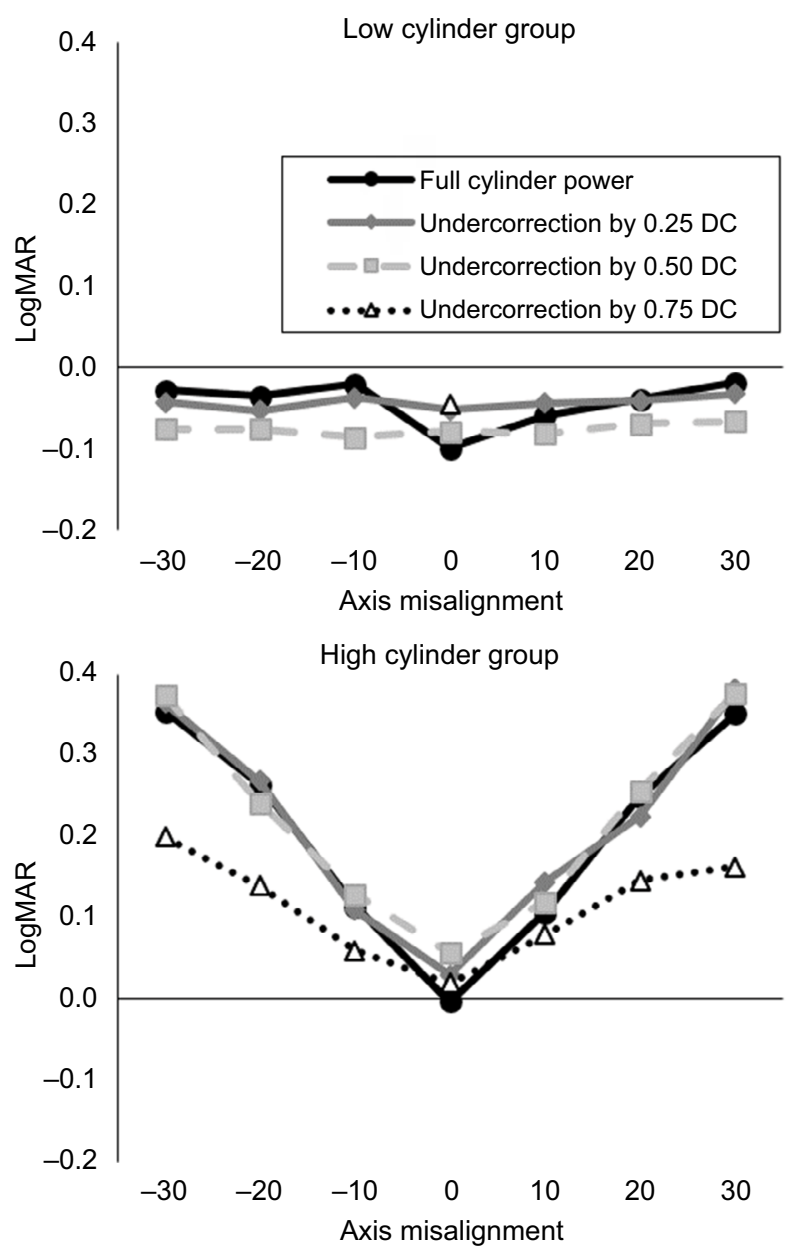

Figure 2 HCVA with different cylinder corrections in the low ( $\leq 0.75 \mathrm{DC})$, medium ( $1.00-1.75 \mathrm{DC})$, and high $(\geq 2.00 \mathrm{DC})$ cylinder groups and in the overall sample. Abbreviation: HCVA, high-contrast visual acuities.
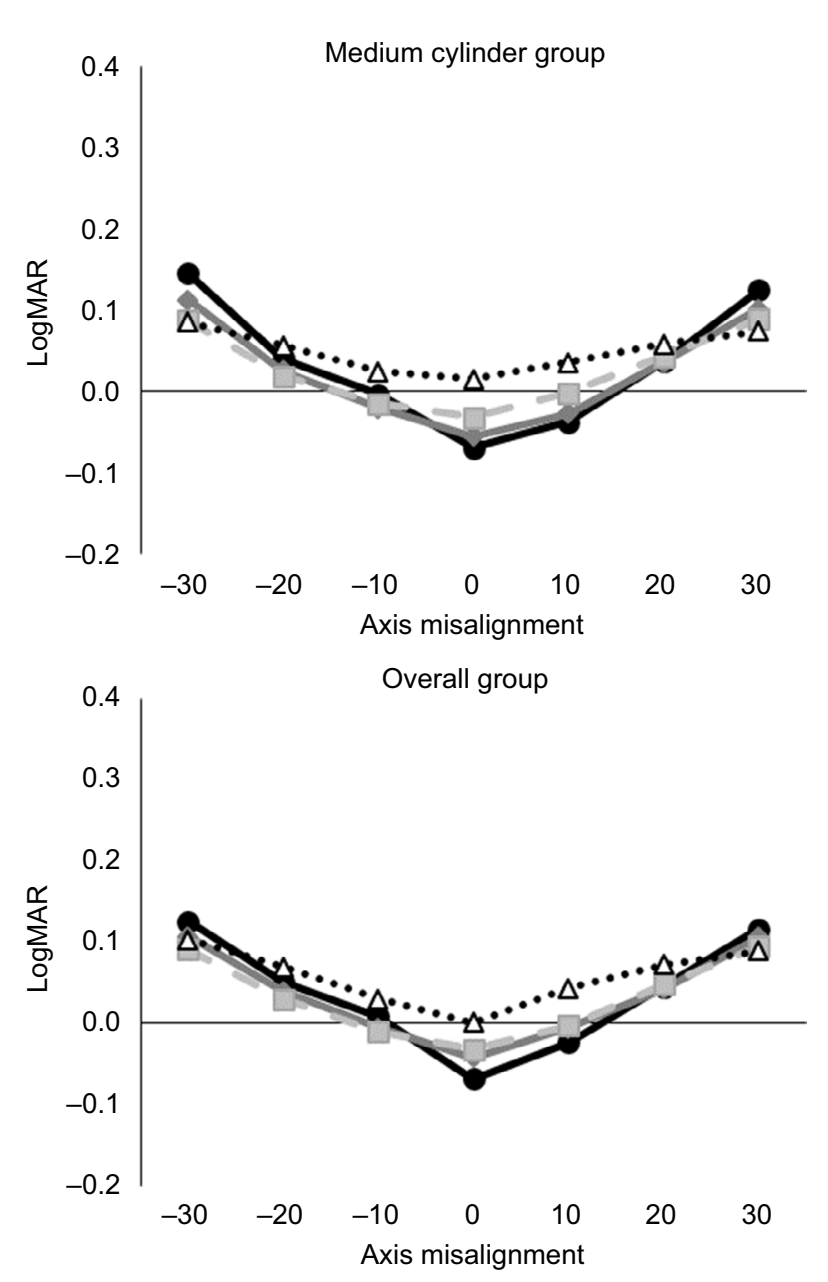

directly comparable to the current study's measures of visual performance. ${ }^{36,37}$

Wolffsohn et al found that each diopter of uncorrected astigmatism caused HCVA loss of 1.5 lines and LCVA loss of 1.4 lines, ${ }^{23}$ while Atchison and Mathur reported losses of 1.7 and 1.85 lines per diopter of uncorrected astigmatism for HCVA and LCVA, respectively. ${ }^{22}$ In comparison, the current study results suggest a less dramatic change, with 0.75 DC undercorrection causing, on average, 0.7 and 0.8 lines of VA loss for HCVA and LCVA, respectively (or $\sim 0.9$ and 1.1 lines per diopter respectively, by extrapolation). However, these studies induced astigmatism at specified axes with spectacle lenses, ${ }^{22,23}$ which in most cases were not the participants' natural cylinder axes. As the measurements in the current study were based on the participants' natural cylinder axes, participant adaptation to astigmatism may have resulted in less VA loss by comparison. Indeed, Vinas et al showed that astigmatic patients are adapted such that their VA is less 

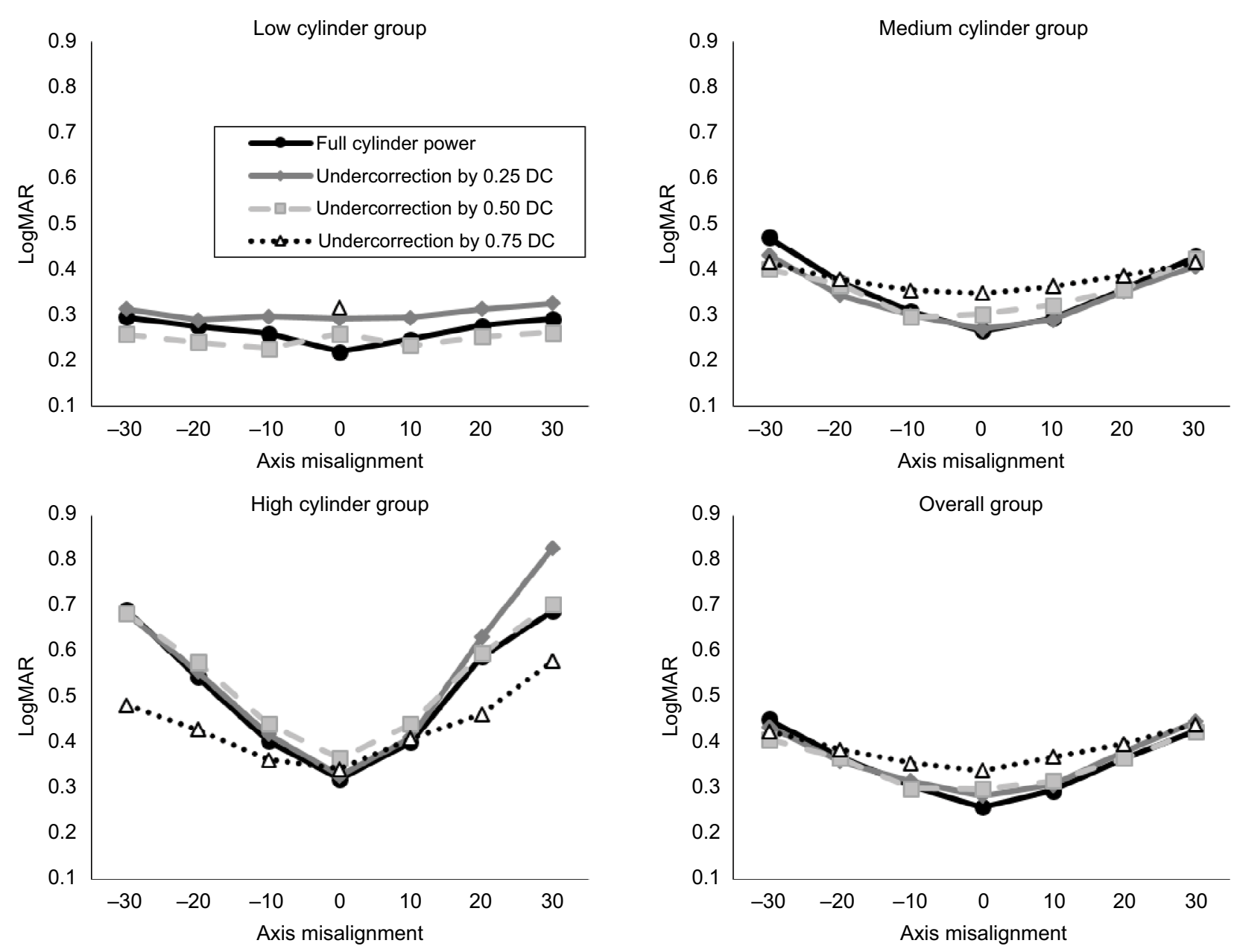

Figure 3 LCVA with different cylinder corrections in the low ( $\leq 0.75 \mathrm{DC})$, medium ( $1.00-1.75 \mathrm{DC})$, and high $(\geq 2.00 \mathrm{DC})$ cylinder groups and in the overall sample. Abbreviation: LCVA, low-contrast visual acuities.

affected when astigmatism is induced at their natural axis, even if their astigmatism is normally corrected. ${ }^{27}$ Figures $2-6$ also demonstrated that undercorrecting cylinder power (while maintaining spherical equivalence) resulted in less sensitivity to axis misalignments than the full cylinder power. Therefore, deliberate undercorrection of cylinder may be of benefit in cases where a toric lens in the desired axis is not available. For example, with daily disposable toric contact lenses where most manufacturers do not have oblique axes available, choosing a lower cylinder power while maintaining spherical equivalence may provide better vision than prescribing the full cylinder power for a patient with oblique astigmatism.

The crossed cylinder effect resulting from a misaligned toric contact (or intraocular) lens is well documented. ${ }^{38-41}$ Alpins demonstrated with vector analysis that a $30^{\circ}$ cylinder misalignment caused loss of half of the astigmatic correction, while a $45^{\circ}$ misalignment caused complete loss of the astigmatic correction. ${ }^{40}$ Tognetto et al confirmed these find- ings using a model simulating an eye with a toric intraocular lens. ${ }^{41}$ Several studies have also investigated the effect of inducing astigmatism at different axes (usually with-the-rule, against-the-rule, and oblique axes); however, these studies generally had a small sample and/or used nonastigmatic participants, and as mentioned previously, induced cylinders at specified axes that may have been different to participants' natural axes. ${ }^{22-25,27}$ The current study aimed to investigate misalignments of cylinder in a way that is more relevant to toric contact lens fitting by testing misalignments of $+30^{\circ}$ to $-30^{\circ}$ on human eyes based on their natural cylinder axes.

Pujol et al reported the greatest decrease in retinal image quality when going from on-axis correction to $5^{\circ}$ of misalignment. ${ }^{26}$ On the other hand, Tognetto et al demonstrated a sigmoidal loss of image quality with lens misalignment, with the most abrupt change occurring between $10^{\circ}$ and $20^{\circ} .^{41}$ Such a shape could be seen for certain groups and variables (most obviously high cylinder group for vision satisfaction, 

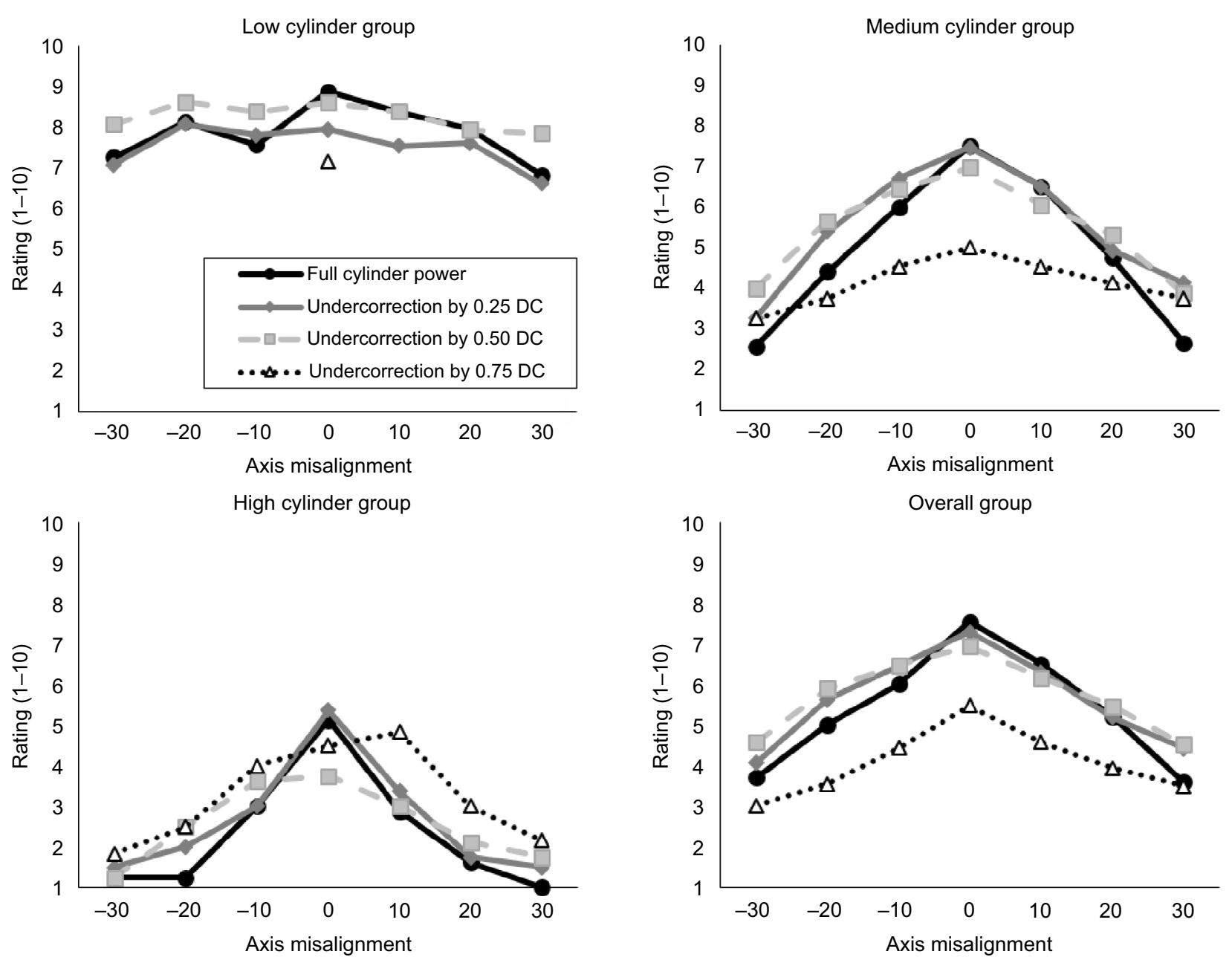

Figure 4 Vision clarity ratings with different cylinder corrections in the low ( $\leq 0.75 \mathrm{DC})$, medium $(1.00-1.75 \mathrm{DC})$, and high $(\geq 2.00 \mathrm{DC})$ cylinder groups, and in the overall sample.

Figure 5), but in general, most other combinations more closely followed either a linear or parabolic pattern. It could also be observed that in the low and overall cylinder groups, $+10^{\circ}$ resulted in better performance than $-10^{\circ}$ (Figures 2-6) . This was an unexpected finding because a positive misalignment always occurred in the anticlockwise direction (rather than toward the horizontal, for example), and furthermore, there was a similar frequency of with-the-rule and againstthe-rule astigmatism. Upon further investigation, this was not a consistent finding but rather an outlier. One low cylinder participant reported very poor performance at $-10^{\circ}$, and this finding was responsible for the decrease in mean results for both the low and overall cylinder group data at $-10^{\circ}$.

Comparing the different cylinder groups in our study suggests different sensitivities to axis misalignments. Based on a clinically significant difference in VA of one line (0.1 $\log$ MAR $),{ }^{32}$ the low cylinder group displayed stable VA between $-30^{\circ}$ and $+30^{\circ}$ misalignments. VA of the medium cylinder group was stable between $-10^{\circ}$ and $+10^{\circ}$ misalignments, while VA of the high cylinder group showed decreased clinical performance when cylinder was misaligned by any tested amount. Based on the above, it might be feasible to reduce the number of SKUs of toric lenses with low cylinder power by up to six times, and toric lenses with medium cylinder power by up to two times. A 2005 survey of eye care practitioners in the United States revealed that less than half are satisfied with a lens rotation of $5^{\circ}$ or more for a patient with $\geq 1.75 \mathrm{DC},{ }^{42}$ which is sensible given the findings of our study. However, those practitioners also considered $10^{\circ}$ to be the maximum acceptable rotation for any toric lens, whereas our data suggest a more flexible allowance may be possible for lower amounts of cylinder. However, it should be noted that $12.5 \%$ of low cylinder participants in this study reported their vision unacceptable with a misalignment of 

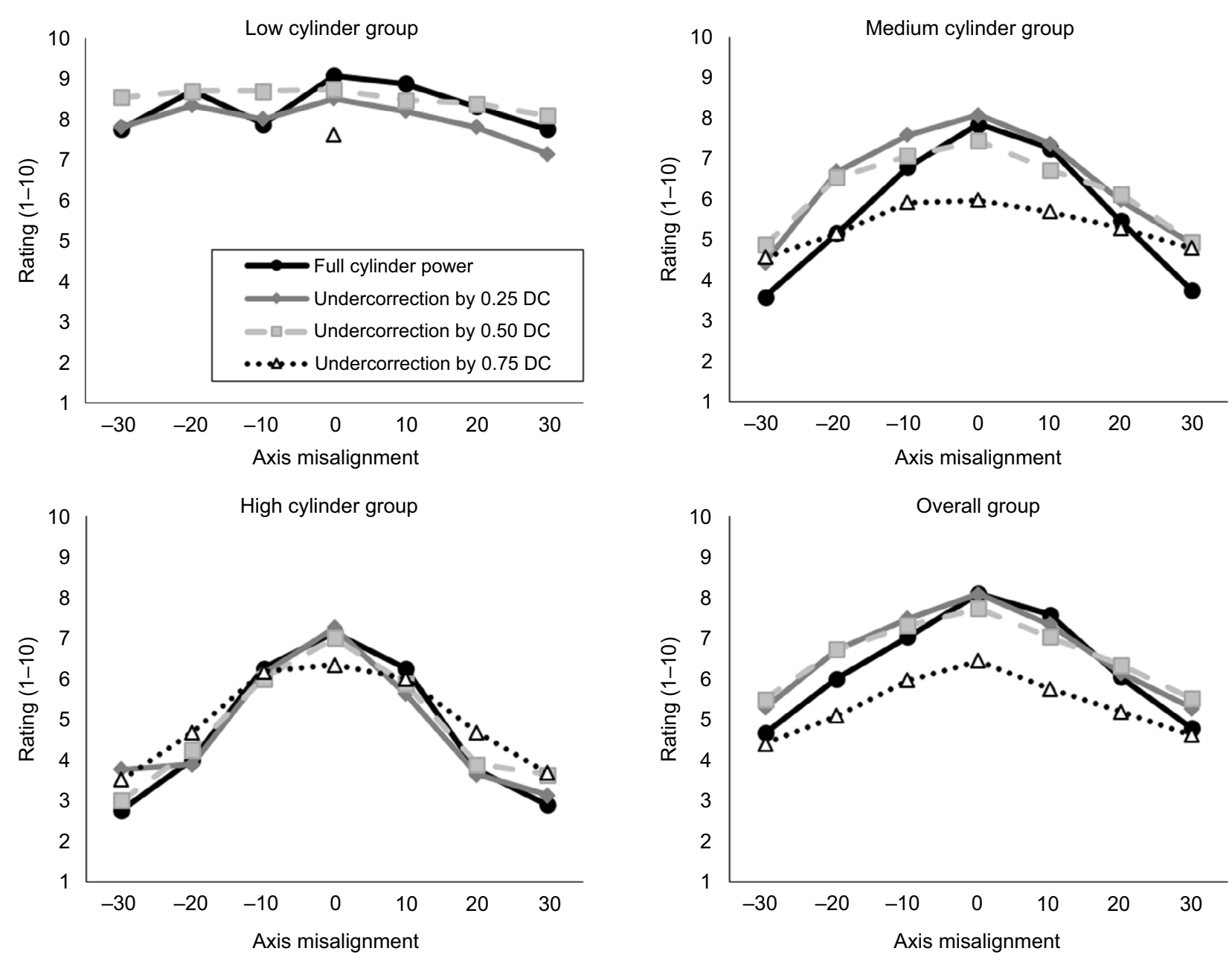

Figure 5 Vision satisfaction ratings with different cylinder corrections in the low ( $\leq 0.75 \mathrm{DC})$, medium (I.00-I.75 DC), and high ( $\geq 2.00 \mathrm{DC})$ cylinder groups and in the overall sample.

$-30^{\circ}$ to $+30^{\circ}$. Therefore, further research is required to determine the balance between reduced SKUs and a potentially increased proportion of astigmatic contact lens wearers who are dissatisfied with their vision. Some aspects to consider in such research include financial concerns and the impact on practitioner confidence with reduced options for lower cylindrical powers. Regardless, these data suggest that toric lenses with high cylinder power should continue to have the complete range of axes available (usually $10^{\circ}$ steps).

Peak visual performance decreased with increasing cylinder, ie, best VAs and subjective ratings in the high cylinder group were worse than the medium cylinder group, which were also worse than the low cylinder group. Based on mean sphere power or spherical equivalent in the subgroups, which was more positive with increasing cylinder, one might have expected the opposite result as positive lenses have a magnifying effect. This may be explained by myopes being less sensitive to blur than nonmyopes. ${ }^{43}$ Another possibility is that spectacle lenses used to correct higher amounts of cylinder cause more meridional magnification, where magnification of the retinal image is unequal in different meridians,,${ }^{44}$ thereby reducing VA and subsequent subjective measures. A similar study design but using contact lenses instead of a phoropter might confirm if this was the case. Additionally, we cannot rule out mild meridional amblyopia in eyes belonging to the high cylinder subgroup, as five out of eight eyes in this group did not reach HCVA of 20/20 when presented with their full correction, though all eyes achieved better than 20/30. The difference in refractive errors and best-corrected visual acuities between the groups is a limitation of this study due to the aforementioned potential confounding effects. However, excluding those participants with higher hyperopia or slightly reduced visual acuity from the high cylinder group would have reduced the sample size significantly and resulted 

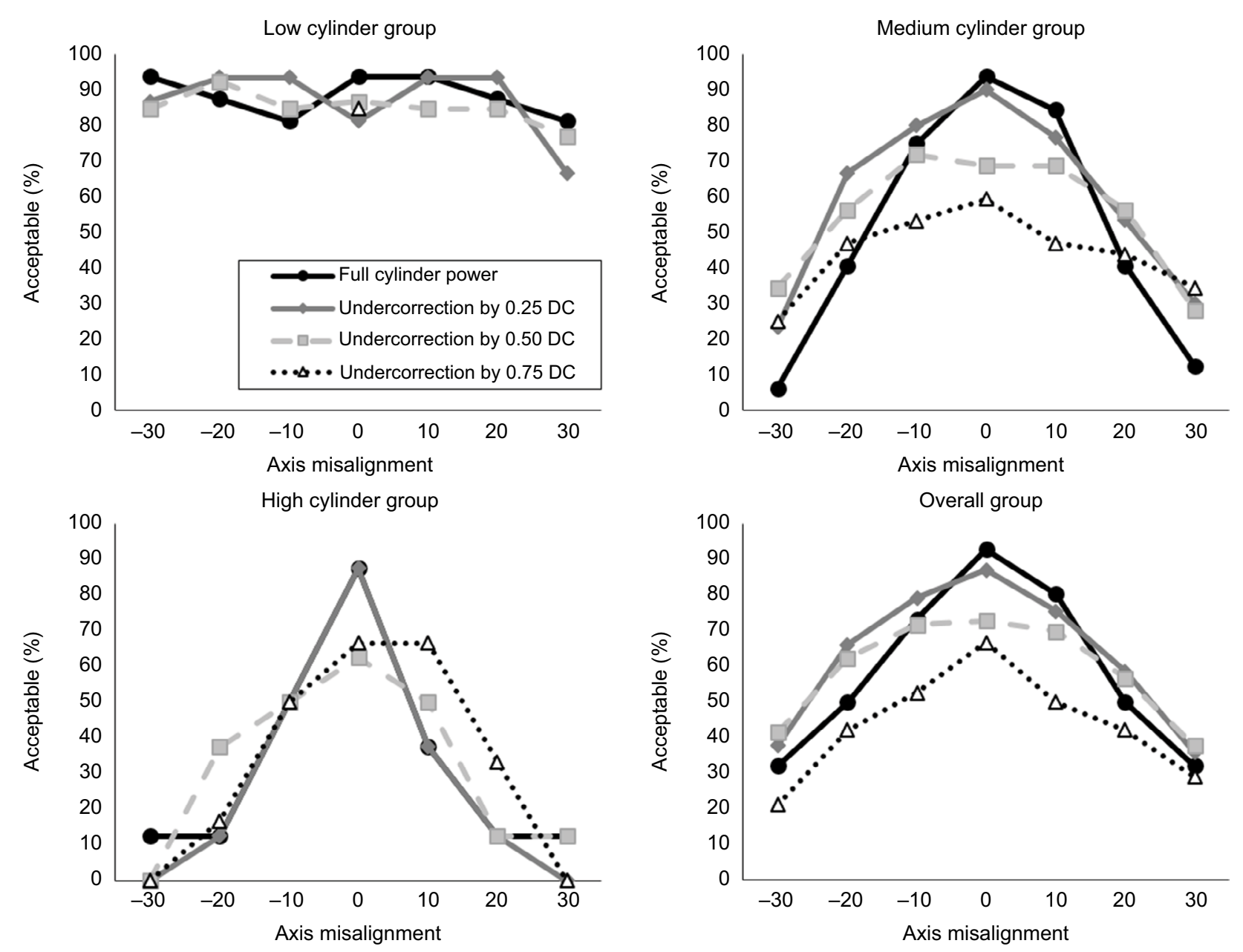

Figure 6 Percentage of participants finding vision acceptable with different cylinder corrections in the low ( $\leq 0.75$ DC), medium (I.00-I.75 DC) and high ( $\geq 2.00 \mathrm{DC})$ cylinder groups, and in the overall sample.

in being unable to make statistical inferences in the group. Furthermore, since the primary objective was to examine relative changes in vision and subjective responses caused by changes in cylinder, rather than the absolute values, we felt that it was valid to include such participants in the analyses.

The use of a phoropter in a monocular setting and the randomized order of the administered clinical procedures in this study may to some extent support the argument that the visual performance data collected from individual eyes is independent, thus facilitating the data analysis at the individual eye level and not at the participant level. Nevertheless, it is important not to forget that the processing of received visual information by the pair of individual eyes occurs at the cortical level. In other words, the data from both eyes of an individual are correlated. To factor this, our statistical methods ensured that correlation of eyes was accounted in the linear mixed model.
A limitation of the study was using a phoropter rather than a trial frame, as head tilt from a participant relative to the phoropter could alter the amount of axis misalignment from the desired amount. However, the phoropter was chosen in preference to the trial frame to eliminate the discomfort of the participant due to the combined weight of a trial frame and lenses when worn for long periods, as well as reducing the time required to complete the study procedures compared to the time required with a trial frame. Furthermore, study investigators regularly checked that each participant remained aligned behind the phoropter while completing the study procedures. Additionally, in the instances where a participant's cylinder power was equal to the tested undercorrection, ie, the participant looked through a spherical lens only, an additional plano lens was not placed in front of the eye, resulting in fewer lens surfaces and potentially changing light transmittance compared to other conditions. However, such an effect would likely be minimal. 
In conclusion, cylinder power can be under corrected by up to 0.50 DC without having a significant effect on HCVA, LCVA, vision clarity, and vision satisfaction. The amount of axis misalignment that can be tolerated is dependent on the cylinder power. These results may have practical ophthalmic applications such as reducing the number of SKUs for toric contact lenses.

\section{Data sharing statement}

The authors do not intend to share data from this study publicly; however, the data are available from the corresponding author upon reasonable request.

\section{Acknowledgments}

The authors would like to acknowledge the clinical team (Ms E Robertson and Ms B Ludlow) and the database management team (Dr T Naduvilath and Ms K Laarakkers) for their invaluable support to run this trial at the Clinical Research Trials Center, Brien Holden Vision Institute, Sydney, Australia. This project was entirely funded by the Brien Holden Vision Institute. Interim findings from this paper were presented at the American Academy of Optometry Conference in Chicago (2017) as poster presentations. The abstracts were published in Optometry \& Vision Science: 1) Fedtke et al. Effect of cylinder power and axis changes on vision in participants with low, medium and high astigmatism. Optom Vis Sci. 2017;94:E-abstract 175395.

2) Tilia et al. Sensitivity of astigmats to changes in cylinder power and axis. Optom Vis Sci. 2017;94:E-abstract 175397.

\section{Disclosure}

The authors report no conflicts of interest in this work.

\section{References}

1. Dirani M, Islam A, Shekar SN, Baird PN. Dominant genetic effects on corneal astigmatism: the genes in myopia (GEM) twin study. Invest Ophthalmol Vis Sci. 2008;49(4):1339-1344.

2. Hammond CJ, Snieder H, Gilbert CE, Spector TD. Genes and environment in refractive error: the twin eye study. Invest Ophthalmol Vis Sci. 2001;42(6):1232-1236.

3. Read SA, Collins MJ, Carney LG. The influence of eyelid morphology on normal corneal shape. Invest Ophthalmol Vis Sci. 2007;48(1):112-119.

4. Lieberman DM, Grierson JW. The lids influence on corneal shape. Cornea. 2000;19(3):336-342.

5. Bagheri A, Farahi A, Guyton DL. Astigmatism induced by simultaneous recession of both horizontal rectus muscles. JAAPOS. 2003;7(1):42-46.

6. Nardi M, Rizzo S, Pellegrini G, Lepri A. Effects of strabismus surgery on corneal topography. J Pediatr Ophthalmol Strabismus. 1997;34(4):244-246.

7. Romero-Jiménez M, Santodomingo-Rubido J, Wolffsohn JS. Keratoconus: a review. Cont Lens Anterior Eye. 2010;33(4):157-166.

8. Kee CS, Hung LF, Qiao-Grider Y, Roorda A, Smith EL. Effects of optically imposed astigmatism on emmetropization in infant monkeys. Invest Ophthalmol Vis Sci. 2004;45(6):1647-1659.
9. Kee C-SU, Hung LI-F, Qiao-Grider Y, Ramamirtham R, Smith EL. Astigmatism in monkeys with experimentally induced myopia or hyperopia. Optom Vis Sci. 2005;82(4):248-260.

10. Dandona R, Dandona L, Naduvilath TJ, et al. Refractive errors in an urban population in southern India: the Andhra Pradesh eye disease study. Invest Ophthalmol Vis Sci. 1999;40(12):2810-2818.

11. Nemeth G, Szalai E, Berta A, Modis L. Astigmatism prevalence and biometric analysis in normal population. Eur J Ophthalmol. 2013;23(6):779-783.

12. Katz J, Tielsch JM, Sommer A. Prevalence and risk factors for refractive errors in an adult inner city population. Invest Ophthalmol Vis Sci. 1997;38(2):334-340.

13. Hashemi H, Rezvan F, Yekta AA, et al. The prevalence of astigmatism and its determinants in a rural population of Iran: the "Nooravaran Salamat" mobile eye clinic experience. Middle East Afr J Ophthalmol. 2014;21:175-181.

14. Vitale S, Ellwein L, Cotch MF, Ferris FL, Sperduto R. Prevalence of refractive error in the United States, 1999-2004. Arch Ophthalmol. 2008;126(8):1111-1119.

15. Williams KM, Verhoeven VJ, Cumberland P, et al. Prevalence of refractive error in Europe: the European Eye Epidemiology (E(3)) Consortium. Eur J Epidemiol. 2015;30(4):305-315.

16. Young G, Sulley A, Hunt C. Prevalence of astigmatism in relation to soft contact lens fitting. Eye Contact Lens. 2011;37(1):20-25.

17. Shih YF, Hsiao CK, Tung YL, et al. The prevalence of astigmatism in Taiwan school children. Optom Vis Sci. 2004;81(2):94-98.

18. Tong L, Saw S-MEI, Carkeet A, et al. Prevalence rates and epidemiological risk factors for astigmatism in Singapore school children. Optom Vis Sci. 2002;79(9):606-613.

19. Tien YW, Foster PJ, Hee J, et al. Prevalence and risk factors for refractive errors in adult Chinese in Singapore. Invest Ophthalmol Vis Sci. 2004;41:2486-2494.

20. Morgan PB, Woods CA, Tranoudis IG, et al. International contact lens prescribing in 2016. Contact Lens Spectrum. 2017;32:30-35.

21. Leube A, Kovats I, Wahl S, Sickenberger W. Axis-free correction of astigmatism using bifocal soft contact lenses. Cont Lens Anterior Eye. 2017;40(6):394-400.

22. Atchison DA, Mathur A. Visual acuity with astigmatic blur. Optom Vis Sci. 2011;88(7):E798-E805.

23. Wolffsohn JS, Bhogal G, Shah S. Effect of uncorrected astigmatism on vision. J Cataract Refract Surg. 2011;37(3):454-460.

24. Kobashi H, Kamiya K, Shimizu K, Kawamorita T, Uozato H. Effect of axis orientation on visual performance in astigmatic eyes. $J$ Cataract Refract Surg. 2012;38(8):1352-1359.

25. Remón L, Tornel M, Furlan WD. Visual acuity in simple myopic astigmatism: influence of cylinder axis. Optom Vis Sci. 2006;83(5):311-315.

26. Pujol J, Arjona M, Arasa J, Badia V. Influence of amount and changes in axis of astigmatism on retinal image quality. J Opt Soc Am A Opt Image Sci Vis. 1998;15(9):2514-2521.

27. Vinas M, de Gracia P, Dorronsoro C, et al. Astigmatism impact on visual performance: meridional and adaptational effects. Optom Vis Sci. 2013;90(12):1430-1442.

28. Watanabe K, Negishi K, Kawai M, et al. Effect of experimentally induced astigmatism on functional, conventional, and low-contrast visual acuity. J Refract Surg. 2013;29(1):19-25.

29. Singh A, Pesala V, Garg P, Bharadwaj SR. Relation between uncorrected astigmatism and visual acuity in pseudophakia. Optom Vis Sci. 2013;90(4):378-384.

30. Ohlendorf A, Tabernero J, Schaeffel F. Visual acuity with simulated and real astigmatic defocus. Optom Vis Sci. 2011;88(5):562-569.

31. Fedtke C, Ehrmann K, Falk D, Bakaraju RC, Holden BA. The BHVIEyeMapper: peripheral refraction and aberration profiles. Optom Vis Sci. 2014;91(10):1199-1207.

32. Rosser DA, Cousens SN, Murdoch IE, Fitzke FW, Laidlaw DA. How sensitive to clinical change are ETDRS $\log$ MAR visual acuity measurements? Invest Ophthalmol Vis Sci. 2003;44(8):3278-3281. 
33. Villegas EA, Alcón E, Artal P. Minimum amount of astigmatism that should be corrected. J Cataract Refract Surg. 2014;40(1):13-19.

34. Dabkowski JA, Roach MP, Begley CG. Soft toric versus spherical contact lenses in myopes with low astigmatism. Int Contact Lens Clin. 1992;19(11-12):252-256.

35. Richdale K, Berntsen DA, Mack CJ, Merchea MM, Barr JT. Visual acuity with spherical and toric soft contact lenses in low- to moderateastigmatic eyes. Optom Vis Sci. 2007;84(10):969-975.

36. Artal P, Green DG, Iglesias I, López-Gil N. Double-pass measurements of the retinal-image quality with unequal entrance and exit pupil sizes and the reversibility of the eye's optical system. J Opt Soc Am A Opt Image Sci Vis. 1995;12(10):2358-2366.

37. Artal P, Marcos S, Williams DR, Navarro R, Navarro R, Aberrations O. Odd aberrations and double-pass measurements of retinal image quality. J Opt Soc Am A Opt Image Sci Vis. 1995;12(2):195-201.
38. Snyder C. A review and discussion of crossed cylinder effects and over-refractions with toric soft contact lenses. Int Contact Lens Clin. 1989;16(4):113-118.

39. Holden BA. The principles and practice of correcting astigmatism with soft contact lenses. Aust J Optom. 1975;58(8):279-299.

40. Alpins NA. Vector analysis of astigmatism changes by flattening, steepening, and torque. J Cataract Refract Surg. 1997;23(10):1503-1514.

41. Tognetto D, Perrotta AA, Bauci F, et al. Quality of images with toric intraocular lenses. J Cataract Refract Surg. 2018;44(3):376-381.

42. Hickson-Curran S, Dias L. Toric soft contact lenses: where are we now? Optician. 2006;231:14-18.

43. Rosenfield M, Abraham-Cohen JA. Blur sensitivity in myopes. Optom Vis Sci. 1999;76(5):303-307.

44. Guyton DL. Prescribing cylinders: the problem of distortion. Surv Ophthalmol. 1977;22(3):177-188.
Clinical Optometry

\section{Publish your work in this journal}

Clinical Optometry is an international, peer-reviewed, open access journal publishing original research, basic science, clinical and epidemiological studies, reviews and evaluations on clinical optometry. All aspects of patient care are addressed within the journal as well as the practice of optometry including economic and business analyses. Basic and clinical

Submit your manuscript here: https://www.dovepress.com/clinical-optometry-journal
Dovepress

research papers are published that cover all aspects of optics, refraction and its application to the theory and practice of optometry. The manuscript management system is completely online and includes a very quick and fair peer-review system, which is all easy to use. Visit http://www.dovepress. com/testimonials.php to read real quotes from published authors. 